\title{
ESTUDO SOBRE AS CONDIÇÕES DE ACESSIBILIDADE NAS RAMPAS E NAS ESCADAS DA ÁREA EXTERNA DO CAMPUS DA UFRPE, EM GARANHUNS/PE.
}

\author{
Amanda Cybelle Fernandes Cavalcanti ${ }^{1}$
}

Maria do Carmo de Albuquerque Braga ${ }^{2}$

Amanda Mayara da Costa Lima Santos ${ }^{3}$

\begin{abstract}
RESUMO
A acessibilidade é um direito garantido constitucionalmente e como tal compreende dimensões físicas e sociais. Diz respeito à facilidade de acesso por qualquer pessoa, aos ambientes físicos, aos bens e serviços, à informação. A partir das propostas de interiorização das universidades públicas em várias regiões do país, pelos governos federais e estaduais, a Universidade Federal Rural de Pernambuco, inaugurou em Garanhuns, uma Unidade Acadêmica, em 2005. E com esta nova implantação, foram construídos blocos com salas de aulas, estruturas administrativas, acessos e estacionamentos. Assim sendo, o objetivo deste trabalho foi analisar as condições de Acessibilidade nas áreas externas do campus da UFRPE, em Garanhuns/PE. A metodologia utilizada privilegiou dois aspectos: observação e análise comparativa. Para observação foram verificados os pontos de acesso trabalhados como escadas e rampas. Para a análise foi feito um comparativo com a legislação em vigor, quando se constatou que $30 \%$ das rampas analisadas possuem uma inclinação menor que o recomendado, além de erros construtivos, enquanto que nas escadas o principal erro está nos espelhos, com altura mínima de $8 \mathrm{~cm}$ e máxima de $21 \mathrm{~cm}$. Assim, a partir dos problemas identificados, mesmo sabendo que parte deles é de alto custo para os devidos ajustes, pode-se evitar que se repitam nas novas unidades acadêmicas a serem implantadas nas demais regiões do estado de Pernambuco.
\end{abstract}

\footnotetext{
${ }^{1}$ Graduanda em Agronomia, Universidade Federal Rural de Pernambuco - Unidade Acadêmica de Garanhuns. E-mail: amanda_cybelles2@hotmail.com;

${ }^{1}$ Professora Adjunta da Universidade Federal Rural de Pernambuco - Unidade Acadêmica de Garanhuns. E-mail: mariabraga77@gmail.com.

${ }^{3}$ Graduanda em Agronomia, Universidade Federal Rural de Pernambuco - Unidade Acadêmica de Garanhuns. E-mail: amanda_mayara2@hotmail.com.
} 


\title{
STUDY ON THE CONDITIONS OF ACCESS RAMP ON THE STAIRS AND AREA OUTSIDE OF CAMPUS UFRPE, IN GARANHUNS / PE.
}

\begin{abstract}
Accessibility is a right guaranteed in the Constitution and as such comprises physical and social dimensions. Respect to ease of access by any person to physical environments, goods and services, information. From the proposed internalization of public universities in various regions of the country, the Federal and State governments, the Federal Rural University of Pernambuco, inaugurated in Garanhuns, an Academic Unit in 2005 and with this new deployment, blocks were built with rooms classes, administrative structures, access and parking. Therefore, the aim of this study was to analyze the conditions of accessibility in the external areas of the campus UFRPE in Garanhuns / PE. The methodology favored two things: observation and comparison. To observe access points worked as stairs and ramps were checked. For a comparative analysis was made with the legislation in force when it was found that $30 \%$ of analyzed ramps have a slope lower than recommended, plus constructive errors, while the stairs is the main error in the mirrors, with a minimum height of $8 \mathrm{~cm}$ and a maximum of $21 \mathrm{~cm}$. Thus, from the problems identified, even though part of it is very costly for the necessary adjustments, you can avoid repeating the new academic to be implemented in other regions of the state of Pernambuco units.
\end{abstract}

KEY-WORDS: Accessibility. Stairs. Ramps.

\section{ESTUDIO SOBRE LAS CONDICIONES DE RAMPA DE ACCESO EN LAS ESCALERAS Y AREA FUERA DEL CAMPUS UFRPE, EN GARANHUNS / PE.}

\begin{abstract}
RESUMEN
La accesibilidad es un derecho garantizado en la Constitución y, como tal, comprende las dimensiones físicas y sociales. Respecto a la facilidad de acceso de cualquier persona a entornos físicos, bienes y servicios, información. Desde la internalización propuesta de las universidades públicas en las diversas regiones del país, los gobiernos federales y estatales, la Universidad Federal Rural de Pernambuco, inaugurado en Garanhuns, una Unidad Académica en 2005 y con esta nueva implementación, los bloques fueron construidos con habitaciones clases, estructuras administrativas, acceso y aparcamiento. Por lo tanto, el objetivo de este estudio fue analizar las condiciones de accesibilidad en las áreas externas del campus UFRPE en Garanhuns / PE. La metodología favoreció dos cosas: la observación y la comparación. Para observar los puntos de acceso trabajado como escaleras y rampas se verificaron. Para un análisis comparativo se realizó con la legislación vigente, cuando se
\end{abstract}




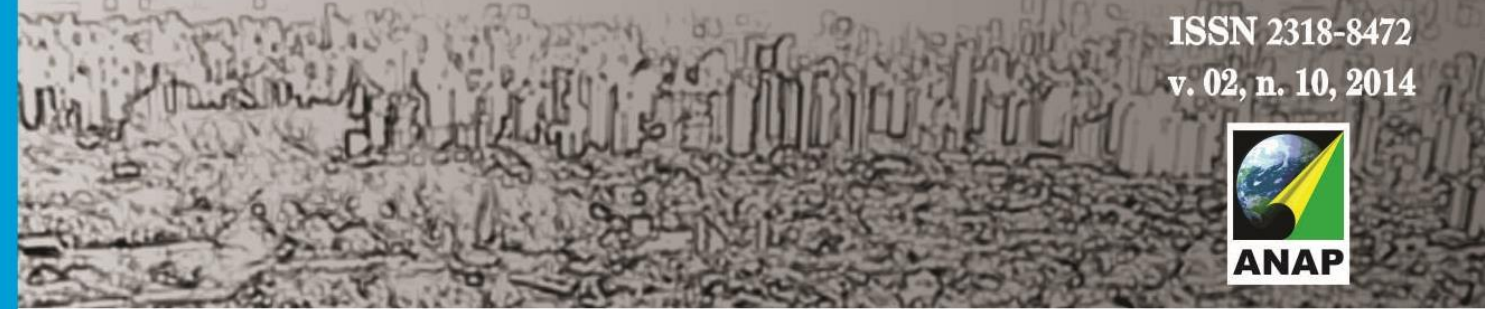

Revista Nacional de

Gerenciamento de Cidades

encontró que el 30\% de las rampas analizadas tiene una pendiente inferior al recomendado, más los errores constructivos, mientras que la escalera es el principal error en los espejos, con una altura mínima de $8 \mathrm{~cm}$ y un máximo de $21 \mathrm{~cm}$. Por lo tanto, a partir de los problemas identificados, a pesar de que parte de ella es muy costoso para los ajustes necesarios, se puede evitar la repetición de la nueva académica para ser implementado en otras regiones del estado de las unidades de Pernambuco.

PALABRAS-CLAVE: Acessibilidad. Escaleras. Rampas.

\section{INTRODUÇÃO}

A acessibilidade é um direito garantido constitucionalmente e como tal compreende dimensões físicas e sociais. Trata da possibilidade dos indivíduos terem acesso e fazer uso de um ambiente ou de um equipamento, deforma livre, independente. Os cidadãos que possuem deficiência e mobilidade reduzida têm que ter a garantia, ao longo da vida, da igualdade de oportunidades no acesso à ambiente, bens e serviços. Para tal foram sancionadas leis, normas e regulamentos que garantem tais direitos (BRASIL, 2003).

As edificações em geral, e principalmente aquelas que abrigam as instituições públicas, devem oferecer a acessibilidade, pois a falta dela prejudica o convívio e o direito de ir e vir das pessoas que possuem mobilidade reduzida, sendo esta temporária ou não. As barreiras físicas podem interferir na utilização dos edifícios, pois sua presença impossibilita que o cidadão usufrua do espaço, e até mesmo, chegue ao seu destino final.

Um dos espaços cujas edificações necessitam oferecer acessibilidade são as universidades públicas, pois estas contribuem para a formação e informação das pessoas em geral. Em alguns casos, essa contribuição, que é um direito de todos, torna-se limitada, impossibilitando que as pessoas com deficiência física utilizem de forma adequada as instalações, negando assim o seu direito a informação.

A partir das propostas de interiorização das universidades públicas no pais, pelos governos federal e estadual, a Universidade Federal Rural de Pernambuco inaugurou, em Garanhuns, uma Unidade Acadêmica, em 2005. Para tal implantação foi necessária a construção de blocos com salas de aulas, estruturas 


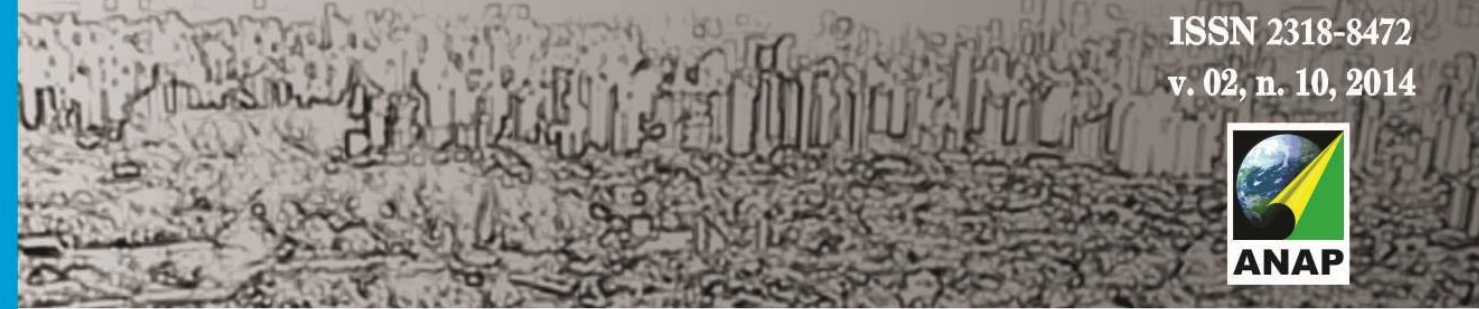

Revista Nacional de

Gerenciamento de Cidades

administrativas, acessos e estacionamentos, possibilitando assim seu uso por todos os cidadãos da região. Assim, foi realizada uma pesquisa PIBIC, em 2013-2014, para analisar as condições de acessibilidade na área externa do campus da UFRPE, em Garanhuns, o que serviu de base para o presente artigo. Neste, o objetivo principal foi o de analisar as condições de acessibilidade da área externa do mencionado campus da UFRPE, em Garanhuns, no que se refere às escadas e as rampas nele existente, com a intenção de verificar se tais elementos estão de acordo com as exigências da legislação, bem como contribuir para a promoção da igualdade de direitos e justiça social, no acesso à informação e a formação profissional para as pessoas da região.

\section{DESENVOLVIMENTO}

Para identificação da acessibilidade na Unidade Acadêmica de Garanhuns UAG foi realizada uma revisão de literatura, embasada em trabalhos sobre o tema. Foram verificadas a utilização e execução de acessibilidade na UAG, tendo como referência as normas, leis e regulamentos que tratam da acessibilidade, entre elas:

- ABNT: NBR 9050/04 - Estabelece critérios e parâmetros técnicos a serem observados nas edificações para possuírem condições de acessibilidade;

- Lei n 7.405/85 - Colocação do "Símbolo Internacional de Acesso" em todos os locais que permitam a utilização por pessoas portadoras de deficiência;

- Lei $n^{\circ}$ 10.098/00 - Normas para a promoção da acessibilidade das pessoas portadoras de deficiência ou com mobilidade reduzida;

- Decreto № 5296/04 - Condição para utilização dos espaços mobiliários, das edificações, dos serviços de transporte e informação, por pessoa portadora de deficiência ou com mobilidade reduzida;

Após o estudo teórico, foi feita a visita de campo, quando se observou se a legislação estudada estava sendo obedecida na área externa da UAG, considerando 


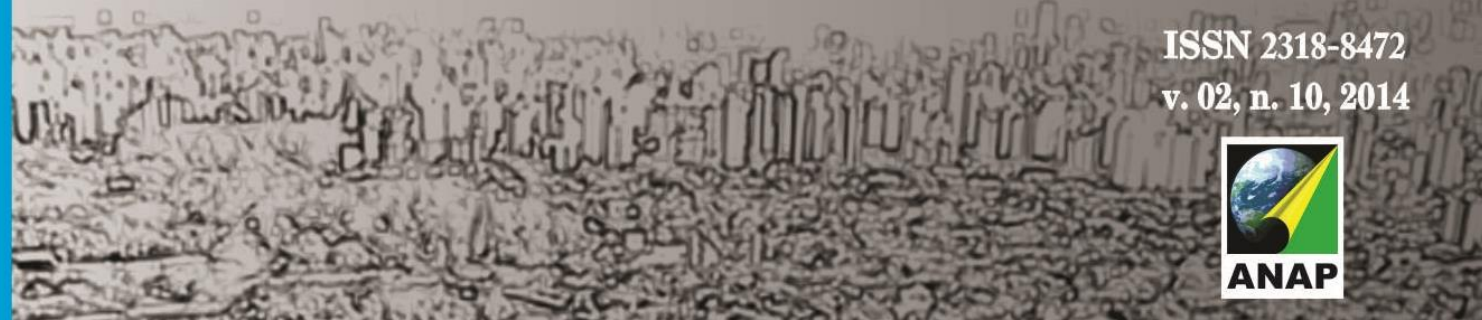

Revista Nacional de

Gerenciamento de Cidades

elementos construtivos como escadas e rampas. Para tal, alguns materiais foram utilizados como auxilio, sendo eles:

-Trena: para auxiliar na medição das rampas, (comprimento e largura), e das escadas, (espelho e piso).

-Caderneta: para demarcar os locais visitados, anotar as medidas e fazer os cálculos dos ângulos das rampas, além das observações em geral.

-Câmera: para registro dos pontos de acesso visitados.

-Mapa: para melhor identificação dos pontos visitados.

Como resultado da visita in loco e para melhor entendimento das observações, foi elaborado um mapa indicativo da localização dos pontos observados (Figura 1). No mapa, a localização das escadas estão apresentadas em vermelho enquanto a localização das rampas em azul.

Figura1: Mapeamento das rampas e escadas.

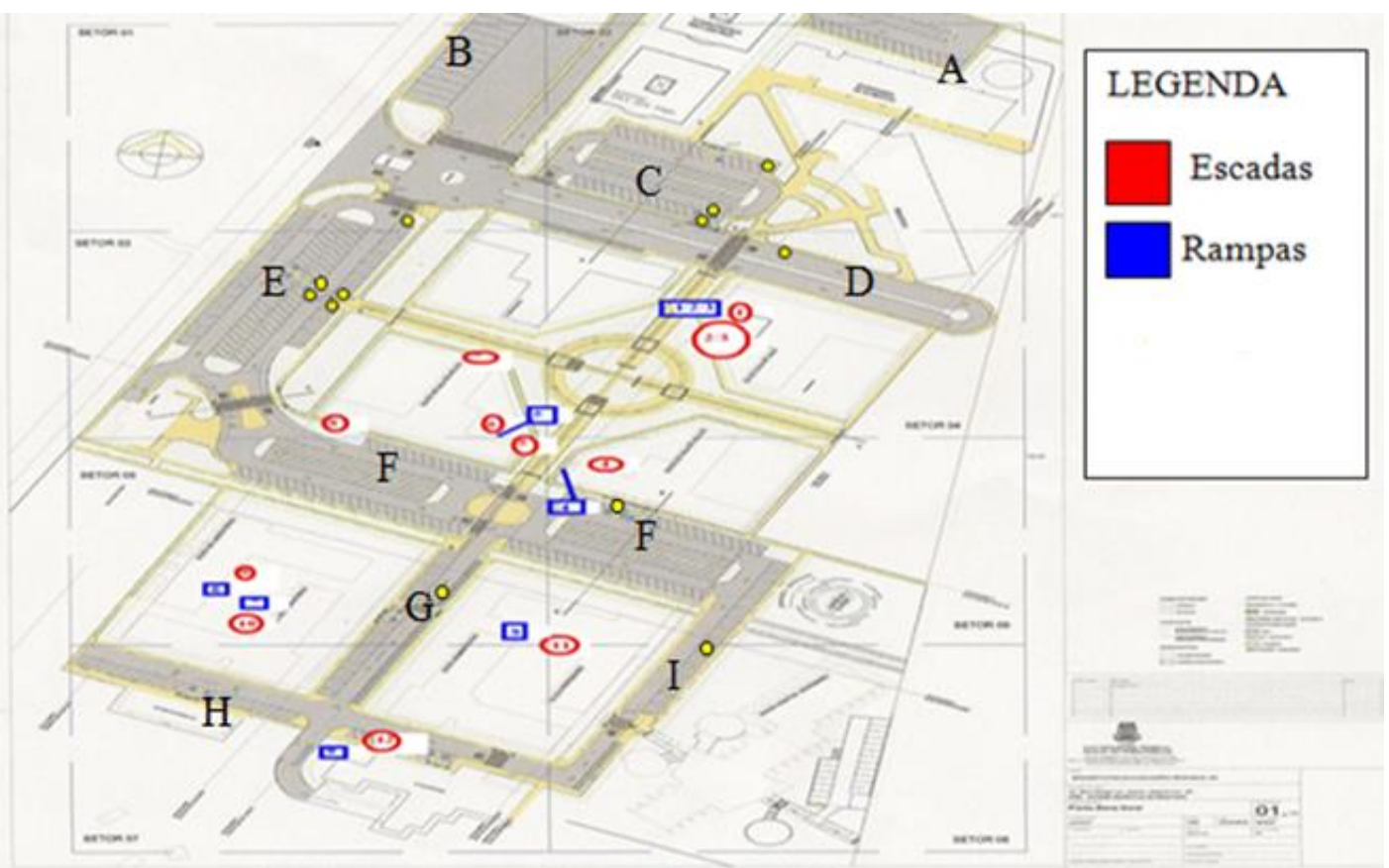

Fonte: EQUIPE DE BOLSISTAS E VOLUNTÁRIOS. GARANHUNS, 2013/2014. 
Revista Nacional de

Gerenciamento de Cidades

Para o levantamento in loco, foi tomada como referência a legislação acima explicitada, seguindo os parâmetros a seguir:

1 - As rampas de acesso devem ter inclinação máxima de 8,33\%, largura livre mínima recomendável em rotas acessíveis é de $1,50 \mathrm{~m}$, sendo o mínimo admissível $1,20 \mathrm{~m}$. Quando não houver paredes laterais as rampas devem incorporar guias de balizamento com altura mínima de $0,05 \mathrm{~m}$, instaladas ou construídas nos limites da largura da rampa e na projeção dos guarda-corpos. No início e no término da rampa devem ser previstos patamares com dimensão longitudinal mínima recomendável de 1,50m, sendo o mínimo admissível 1,20m, além da área de circulação. (ABNT/NBR 9050,2004)

2 - As escadas devem ter uma profundidade de piso que deve estar entre 28 e $32 \mathrm{~cm}$, a altura do espelho deve medir entre 16 e $18 \mathrm{~cm}$, a largura mínima estabelecida é de $1,20 \mathrm{~m}$ e ocorrência de corrimão e guarda corpos. (ABNT/NBR 9050,2004)

Analisando o que recomenda a legislação e os pontos visitados observou-se que das dez rampas visitadas, cerca de $70 \%$ destas estavam de acordo com os padrões de acessibilidade. $O$ valor encontrado para variação de ângulo mínimo foi de 3,8\%e o máximo de $6,6 \%$, não ultrapassando um ângulo maior que 8,33\% como recomenda a $A B N T$. Já os $30 \%$ das rampas que não respeitavam os parâmetros apresentaram ângulos de inclinação com valores extremamente altos como $57,6 \%$; $21,9 \% ; 10,2 \%$, e ainda existindo rampas que não levam a lugar algum (Figura 2).

Figura2: Rampa sem corrimão, guarda corpo, piso tátil e piso direcional.

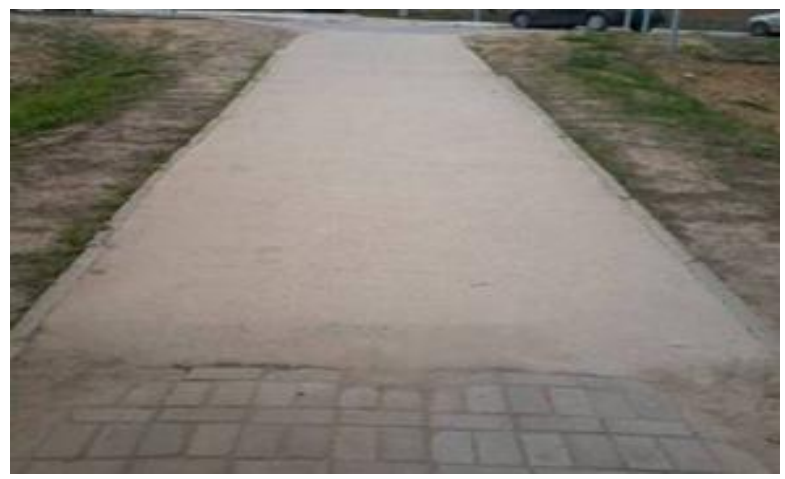

Fonte: EQUIPE DE BOLSISTAS E VOLUNTÁRIOS. GARANHUNS, 2013/2014. 


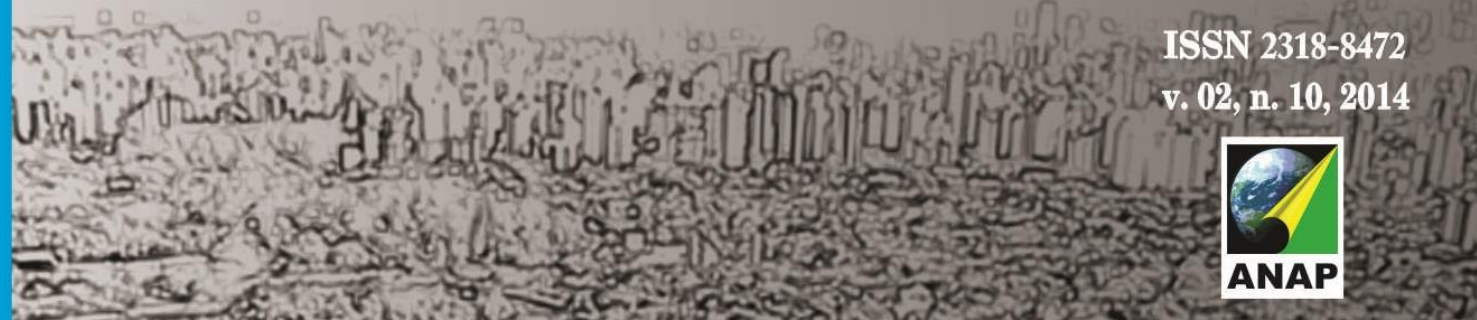

Revista Nacional de

Gerenciamento de Cidades

Grafico1: Distribuição dos espelhos

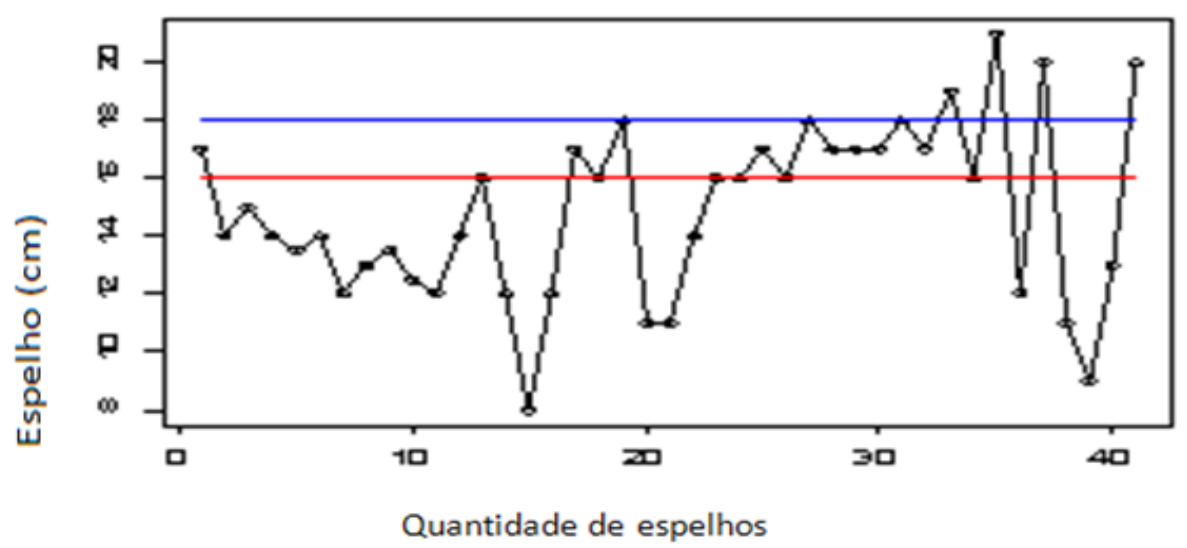

Fonte: EQUIPE DE BOLSISTAS E VOLUNTÁRIOS. GARANHUNS, 2013/2014.

No gráfico 2, indicando o comprimento do piso, este deveria situar-se entre $28 \mathrm{~cm}$, indicado pela cor vermelha, e $32 \mathrm{~cm}$, pela cor azul, porém não é bem isso que acontece. Os 29 pisos analisados estão com comprimento mínimo de $22 \mathrm{~cm}$ e máximo de $52 \mathrm{~cm}$.

\section{Gráfico 2: Distribuição dos pisos}

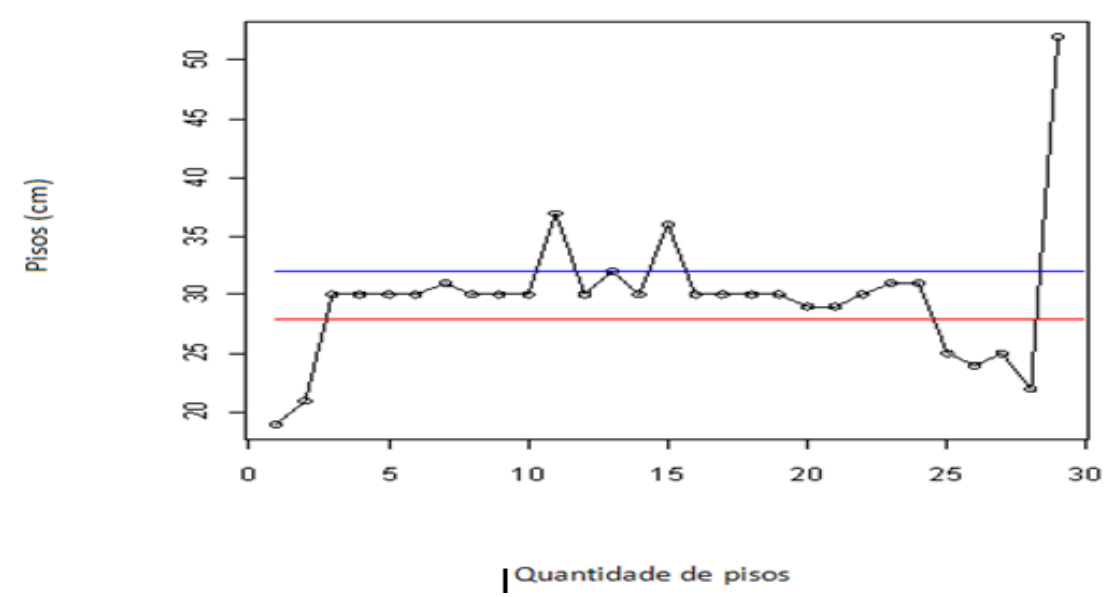

Fonte: EQUIPE DE BOLSISTAS E VOLUNTÁRIOS. GARANHUNS, 2013/2014.

\section{CONCLUSÃO}

Ao observar os espaços externos da Unidade Acadêmica de Garanhuns, notou-se que há a tentativa de promover a acessibilidade em sua construção. O que se observa de fato e que em muitos locais a tentativa não está de 


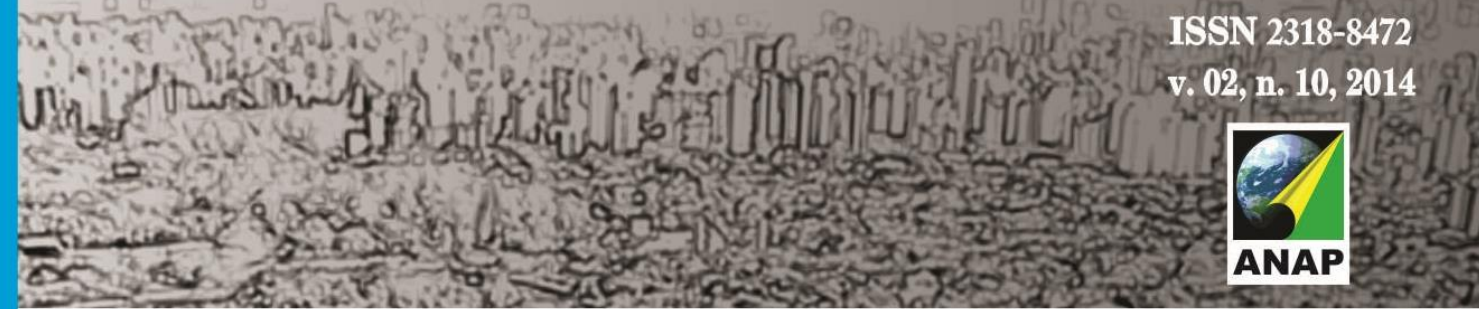

Revista Nacional de

Gerenciamento de Cidades

acordo com o que estabelece a legislação em vigor. Pode-se dizer que de acordo com o que foi analisado em relação às escadas, estas apresentam erros que vão desde alturas mínimas de espelhos como $8 \mathrm{~cm}$, até o extremo de $51 \mathrm{~cm}$. As alturas observadas foram tão variadas e grandes que dificultam até mesmo a circulação de pessoas sem nenhum tipo deficiência.

Em se tratando das rampas, estas apresentaram problemas menores em relação à inclinação, mas não deixando de ser prejudicial aos cadeirantes, que necessitam de um ângulo correto para que não enfrentem dificuldades ao usufruir da possibilidade de obter uma formação acadêmica. Mas, entre os erros graves encontrados, destaca-se uma rampa que não tem finalização, ou seja, não leva seu usuário a lugar algum.

Por fim, conclui-se que os problemas de acessibilidade encontrados na Unidade de Garanhuns são problemas construtivos graves, que para serem corrigidos certamente demandarão novos custos, ou seja, um retrabalho cujo valor poderia estar sendo usado para novos investimentos no campus, mas que se torna fundamental sua execução já que a Universidade sendo um espaço público deve propiciar seu uso a todos os cidadãos, independemente de sexo, cor, idade, nível social, portador ou não de necessidades especiais, temporária ou permanente. Nesse sentido, vale salientar que ao se realizar o trabalho, não se verificou a existência de uma única pessoa com deficiência física fazendo uso do espaço, o que por si só já se torna um fato estranho.

Assim, a partir dos problemas identificados, conclui-se que os mesmos devem ser corrigidos para que a Unidade Acadêmica de Garanhuns possa atender aos cidadãos da região, de acordo com o que estabelece as leis e normas de acessibilidade. Conclui-se ainda que a relevância do trabalho se concentra na intenção deste em fazer com que sejam evitados, que se repitam nas novas unidades acadêmicas a serem implantadas, nas demais regiões do estado de Pernambuco, oportunizando acesso ao conhecimento também às pessoas portadoras de necessidades especiais como estabelece a legislação em vigor. 


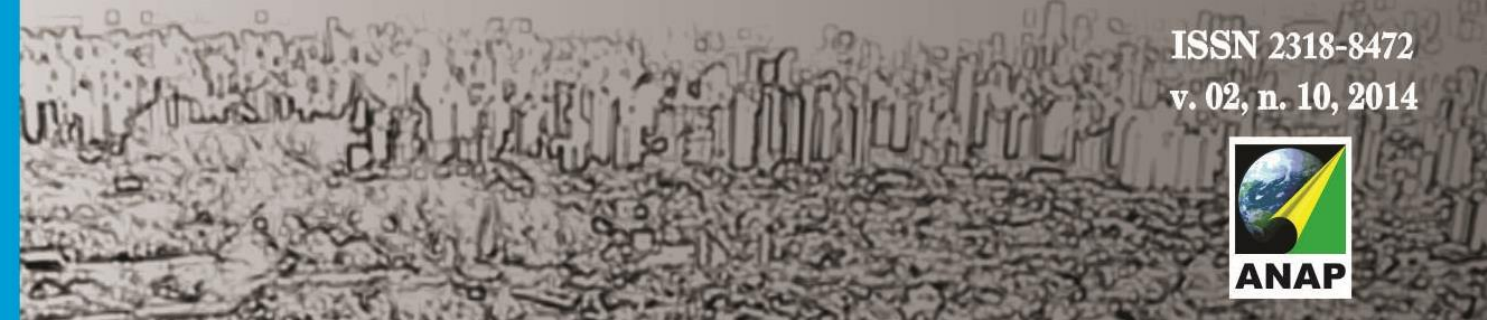

Revista Nacional de

Gerenciamento de Cidades

\section{REFERÊNCIAS}

ABNT- Associação Brasileira de Normas Técnicas - NBR 9050. Acessibilidade a edificações, mobiliário, espaços e equipamentos urbanos. Rio de Janeiro: ABNT, 2004, 42 e 45p.

BRASIL. Constituição da República Federativa do Brasil. 8. ed. São Paulo: Revista dos Tribunais, 2003.

BRASIL. Decreto-lei no. 5296, de 2 de dezembro de 2004. Disponível em: <http://www.planalto.gov.br/ccivil_03/_ato2004-2006/2004decreto/d5296.htm>. Acesso em: 08/09/2014.

BRASIL. Lei no. 7.405, de 12 de novembro de 1985. Disponível em:

<http://www.planalto.gov.br/ccivil_03/leis/1980-1988/L7405.htm>. Acesso em: 09/06/2014.

BRASIL. Lei no. 10.098, de 19 de dezembro de 2000. Disponível em:

<http://portal.mec.gov.br/arquivos/pdf/lei10098.pdf>. Acesso em: 09/06/14. 